\title{
Stefano Aloe
}

\section{Il viaggio in Molise di Marin Drinov e Vikentij Makušev nelle lettere di Giovanni De Rubertis}

\author{
Izvorni znanstveni rad \\ Original scientific paper \\ UDK 39:811.16(450.67)(044)
}

I testi scientifici e privati dei primi slavisti che hanno visitato le colonie slavo-meridionali del Molise sono di interesse per la storia della slavistica italiana. L'attività divulgativa dello storico slavomolisano Giovanni De Rubertis e il viaggio ad Acquaviva Collecroce di due storici slavi, il russo Vikentij Makušev e il bulgaro Marin Drinov nel 1870 diedero avvio a questi studi. La corrispondenza epistolare fra i due eminenti studiosi slavi e l'entusiasta storico dilettante di Acquaviva Collecroce, insieme ad una pagina del Diario di Makušev ed altri materiali inediti permettono di trarre delle considerazioni sulla coscienza etno-linguistica degli slavi molisani nella seconda metà dell'Ottocento; sui loro orientamenti culturali; sulle principali influenze slave giunte fino a loro prima dell'instaurarsi di relazioni strette con l'altra sponda dell'Adriatico.

Ključne riječi: Molizanski (moliški) Hrvati; Giovanni De Rubertis;

Vikentij Makušev; povijest slavistike; hrvatskotalijanski odnosi; Acquavita Collecroce; Kruč

Lo studio ed il confronto dei testi scientifici (articoli) e privati (lettere, diari) dei primi slavisti che visitarono le colonie slavo-meridionali del Molise risulta di un certo interesse per la storia di questa disciplina, e in particolare per lo sviluppo degli studi slavistici internazionali legati all'Italia. Infatti, i primi viaggi slavistici in Molise si svolsero in un periodo (la seconda metà dell'Ottocento) in cui non solo tale filone di studi in Italia era ancora in embrione, ma neanche la slavistica internazionale aveva ancora sviluppato un interesse specifico per l'Italia (vd. Лаптева 2005; Алоэ 2012; Aloe 2013). 
Il punto di partenza fondamentale per tale scoperta e per i suoi sviluppi scientifici viene riconosciuta nell'attività divulgativa dello studioso slavomolisano Giovanni De Rubertis (1813-1889), che sin dagli anni '50 dell'Ottocento aveva cominciato ad adoperarsi affinché il mondo accademico slavo venisse a sapere dell'esistenza delle tre piccole colonie slave, isolate tra i monti del Molise, nel territorio dell'allora Regno delle Due Sicilie. I primi contatti slavistici del De Rubertis sono cosa nota: risalgono alla corrispondenza epistolare intrapresa con lui dal letterato raguseo Medo Pucić e pubblicata dapprima a Zara nel 1856, e poi nella traduzione russa di Osip M. Bodjans'kyj nel $1858 .^{1}$

Fino al 1870, i numerosi viaggi di ricerca degli slavisti avevano trascurato i territori italiani (eccezion fatta per le valli slovenofone del Friuli), e il potenziale interesse storico e archivistico della penisola era stato intuito da pochi. A maggior ragione doveva apparire remotissima la destinazione dell'entroterra molisano, una zona montuosa e depressa, nota per la diffusione del brigantaggio e quasi sprovvista di strade. I primi studiosi ad interessarsi in maniera attiva ai legami fra le città italiane e il mondo slavo furono due storici, il russo Vikentij Makušev e il bulgaro Marin Drinov, che si conobbero casualmente in un archivio di Napoli alla fine del 1869 (Никулина 1974; Минкова 1981, 1986 е 1988). Drinov, destinato di lì a non molto a fondare la scuola storiografica bulgara, era al momento un giovane studioso che si finanziava il soggiorno in Italia stando al servizio di una ricca famiglia patrizia russa. Makušev era invece un allievo di Izmail Sreznevskij all'università di Pietroburgo e aveva ricevuto una borsa di studio per un progetto dottorale che prevedeva la visita dei principali archivi e biblioteche d'Italia alla ricerca di materiali storici legati al mondo slavo. La borsa di studio garantì a Makušev una permanenza in Italia di circa due anni (1869-1871).

Nella primavera del 1870 i due studiosi, divenuti amici, fanno la conoscenza, a Napoli, di un prete di Acquaviva Collecroce, don Paolo Vetta, che li invita in Molise per le festività agresti del primo maggio. Vetta prepara l'arrivo dei due illustri ospiti comunicando la notizia a Giovanni De Rubertis, che nella sua veste di storico locale e di insegnante di ginnasio rappresentava la massima autorità intellettuale del paese. Così, Drinov e Makušev partono da Napoli alla fine di aprile e, compiuto un viaggio faticoso e disagevole di un paio di giorni, raggiungono Acquaviva Collecroce, dove vengono accolti in maniera festosa dalla popolazione e dalle autorità cittadine.

1. G. De Rubertis, 1856; Де Рубертис, 1858. Sulla figura di De Rubertis, vd. Krpan, 1993 : 211-212; Стипчевић, 2007: 287. 
Una pagina del diario di Makušev, vergata parecchi anni dopo, è la testimonianza più significativa delle difficoltà a cui all'epoca andava incontro chi volesse raggiungere queste quasi mitiche colonie slavo-molisane. Makušev scrive, tornando con la memoria a quel viaggio:

(...) „думаю про себя, и невольно приходит мне на мысль корчма в Молизе (в южной Италии), в которой я и спутник мой, М. С. Дринов, провели такую неспокойную и неприятную ночь, отправляясь $\mathrm{k}$ братьям славянам в Воду Живу, - корчма - притон абруццских бандитов, о которых мы достаточно наслышались в Неаполе...“ (Макушев 1881: 16). ${ }^{2}$

In modo più dettagliato, Makušev aveva già descritto quel viaggio nel suo articolo sulle colonie slavo-molisane del 1871 (Макушев 1871: 35-36), evidenziando la sporcizia e il degrado che avevano accolto i viaggiatori a Larino, dove erano stati costretti a trascorrere quella notte inquieta a cui fa riferimento la pagina di diario sopra citata. II giorno successivo, con pioggia battente, fu poi complicato guadare il torrente Biforno in piena, nella totale mancanza di ponti.

Il pericolo del brigantaggio rammentato da Makušev non era una fantasia da viaggiatore suggestionato. Tre mesi dopo la partenza dei due storici da Acquaviva, un nipote del De Rubertis venne rapito mentre attraversava una zona isolata del Molise. Lo stesso De Rubertis ne scriveva con angoscia in una lettera indirizzata al Drinov:

„Al giorno due di questo mese un altro mio nipote, il Signor Carlo Leone di Guglionesi, cognato del padre Romualdo, è stato catturato da' briganti, i quali pretendono pel suo riscatto l'ingente somma di cinquanta mila lire! Pendono le trattative (trattative con i briganti!) e si farà qualunque sagrificio, onde salvare un infelice padre di numerosa famiglia". ${ }^{3}$

2 «...penso fra me e me e senza volerlo mi sovviene quella bettola molisana (in Italia meridionale) dove io ed il mio compagno di viaggio M.S. Drinov passammo una notte molto inquieta e spiacevole, quando eravamo diretti verso i fratelli slavi di Acquaviva; bettola che era un covo dei briganti abruzzesi di cui a Napoli avevamo sentito parlare a sufficienza»...

${ }^{3}$ Lettera di G. De Rubertis a M. Drinov, 11 agosto 1870, НБКМ БИА, ф. 111, арх. ед. 267, л. 5. 
Fortunatamente i due storici slavi giunsero ad Acquaviva incolumi il 29 aprile, per rimanervi solo pochi giorni, ma la visita permise comunque a Makušev di raccogliere una certa quantità di osservazioni e notizie di carattere linguistico, storico, folkloristico che presentò alcuni mesi dopo su una rivista scientifica russa (Макушев 1871: 31-56).

Ho avuto modo di pubblicare in alcuni recenti articoli informazioni su questo viaggio e, in particolare, su quanto finora ho rinvenuto dei brevi scambi epistolari seguiti alla partenza di Makušev e Drinov dal Molise: due lettere di De Rubertis a Makušev ed estratti (in traduzione russa) delle tre lettere da lui indirizzate a Drinov (Алоэ 2012; Aloe 2013). Accludo le tre lettere, ad oggi inedite e custodite presso la Biblioteca Nazionale di Sofia (НБКМ БИА, ф. 111, арх. ед. 267), in appendice al presente articolo.

Sono lettere affettuose, quelle con cui De Rubertis accompagna i due slavisti nei mesi successivi alla loro partenza da Acquaviva. Esse sono un'importante testimonianza del ruolo significativo di quest'uomo: pur di cultura abbastanza modesta, egli, da generoso e appassionato autodidatta, seppe, da una parte, risvegliare nella popolazione slavo-molisana la coscienza della propria appartenenza etno-linguistica e storico-culturale, e dall'altra fu capace di sollecitare interesse scientifico nei confronti di queste piccole comunità che, proprio grazie all'isolamento in cui erano vissute, avevano conservato nelle usanze e nel dialetto tratti peculiari che le legavano agli slavi dell'altra sponda dell'Adriatico. Non è superfluo ricordare che negli anni successivi De Rubertis ebbe modo di accogliere con lo stesso calore e senso dell'ospitalità altri slavisti, tra cui Jan Baudoin de Courtenay e Risto Kovačić (Дуличенко 1981: 95-109; Kovačić 1874; Ковачић 1885: 273-340); e che le sue raccolte di canti popolari e di documenti storici locali rimasero un punto di riferimento per gli studiosi anche dopo la sua morte, sopraggiunta nel 1889. Così, quando agli inizi del XX secolo Miloš Rešetar studiò a fondo le colonie slavo-molisane e dedicò ad esse la prima monografia, ancor oggi fondamentale, il ricordo del De Rubertis era ancora assai vivo e il suo lascito culturale rappresentò una base di primaria importanza per lo slavista dalmata (Rešetar 1911 e 1997).

Tornando alla corrispondenza del De Rubertis con Makušev e Drinov, essa presenta vari spunti di informazione che qui per lo più tralascio, rimandando per essi ai miei precedenti articoli già menzionati. Intendo invece mettere in rilievo alcuni aspetti stilistico-linguistici delle lettere dell'insegnante di Acquaviva Collecroce. Esse nella forma trasmettono il senso di soggezione del De Rubertis di fronte ai due studiosi stranieri, ma anche la sua dignità di storico e di 
conservatore delle tradizioni locali. Ciò che maggiormente lo doveva colpire, portandolo ad una percepibile eccitazione, era la possibilità di comunicare con dei fratelli slavi, come De Rubertis, facendo proprio un costume tipicamente panslavista, era solito chiamare i suoi ospiti provenienti da paesi di lingua slava. L'intera popolazione di Acquaviva Collecroce, preparata al loro arrivo dal De Rubertis, aveva circondato Drinov e Makušev dell'attenzione particolare che poteva essere riservata ai primi slavi stranieri che il paese ricordasse (Макушев 1871: 36-37). Inoltre, il bulgaro e il russo giungevano come rappresentanti di una disciplina, la slavistica, che anche fra i monti del Molise aveva già fatto in tempo a colorarsi di un'aurea idealizzante. II fatto di parlare „la stessa lingua“ (Макушев, 1871: 37; in dialetto slavo-molisano 'na našo/u') spingeva gli entusiasmi verso una vaga passione „panslavista“, peraltro del tutto scevra di connotazioni politiche o territoriali. Si manifestava forse una variante dell'idea comunitaria che è radicata nelle culture slave.

Per questo, non può sorprendere che anche nelle lettere di De Rubertis si affacci il tema della fratellanza slava, con il riflesso di una scrittura affettuosa e familiare che, forse, poté infastidire Makušev, il quale smise presto di rispondere. Singolare il fatto che le lettere siano scritte in italiano, lingua che i due storici slavi conoscevano bene e che anche nei confronti degli abitanti di Acquaviva Collecroce rimaneva, probabilmente, il miglior veicolo di comunicazione e reciproca comprensione: infatti, l'interscambio linguistico che mescolava varianti slave (il dialetto di Acquaviva da parte dei locali, il serbo, il russo e il bulgaro da parte dei due ospiti), poteva sì dimostrare la stretta parentela linguistica con quei remoti visitatori, e per essi costituire oggetto di prime osservazioni dialettogiche, ma non del tutto soddisfare necessità comunicative che andassero al di là di un livello basilare. A giudicare dalle lettere conservate, anche durante il soggiorno ad Acquaviva la comunicazione fra Makušev, Drinov e De Rubertis era avvenuta in lingua italiana, mentre il dialetto slavo locale e il serbo-croato letterario, che De Rubertis masticava in modo approssimativo grazie alle sue precedenti frequentazioni dalmate e serbe, venivano a costituire soltanto l'oggetto del discorso, o fungevano da esempi per gli appunti linguistici di Makušev. Anche nelle lettere De Rubertis ricorre di tanto in tanto ad espressioni e frasi in dialetto slavo-molisano. Tuttavia, già Makušev ebbe modo di accorgersi che le performances scritte del De Rubertis tradivano inesattezze, dovute al passaggio dalla lingua dell'oralità ad una variante letteraria che lui stesso in un certo senso inventava, ibridando il dialetto con quanto conosceva della lingua serbo-croata letteraria della sua epoca. De Rubertis era „poeta, e non linguista“, osservava Makušev nel suo esteso saggio (Макушев 1871: 34). Con questo avvertimento, 
le parti in slavo-molisano delle lettere rimangono comunque un'utile testimonianza. De Rubertis se ne serve nei momenti più emotivi delle sue missive, con lo scopo evidente di gettare un ponte di simpatia e intimità con i suoi corrispondenti. Così, nella lettera indirizzata a Makušev il 28 maggio 1870, una sua tirata appassionata sulla fratellanza slava si conclude con parole del dialetto:

„Oh gli Slavi, checchè ne dica l'Etnografo Sig. Vegezzi-Ruscalla, si amano di vero cuore; ed essi soli si scambiano il saluto col dolce nome di fratelli, (brat)! Oh se gli Slavi formassero una sola nazione, una sola famiglia!.. Ma i destini de' popoli si maturano ne' disegni di Dio! Rimettiamoci adunque alla Divina Provvidenza = Neka cini Bog!“ (Алоэ 2012: 24).

E la seconda ed ultima lettera indirizzata a Makušev si conclude con un post scriptum dialettale dal tono scherzoso, nel quale De Rubertis chiede notizie di Drinov, che non gli scrive:

„E Drinoff? Ni govere, ni piša! Šta čini? Zaspe! Blago njemu! Kada mu pîsaš, potežimu uše! Zdravo" (Алоэ 2012: 27).

L'aspetto marcatamente paratattico delle frasi ed il ricorso a fraseologismi sembrano dimostrare una certa goffaggine nel riportare sulla carta quella parlata, priva di una propria tradizione letteraria. Certo, vi si può leggere anche il desiderio di offrire allo studioso russo un repertorio fraseologico comprensibile e utile ai fini della ricerca (interessante, per esempio, il passaggio alla forma del 'tu', come unica presente nel dialetto, di fronte al 'lei' usato impeccabilmente in italiano). Ma anche l'uso incerto dei segni diacritici, modellati sulle norme croate, tradisce una ricerca di equilibrio fra specificità dialettale e standard linguistico che nel complesso risulta poco coerente.

Maggiori sono gli inserti slavo-molisani nelle lettere a Marin Drinov. La prima lettera, in particolare („Vodaživa, 24 lipanj 1870“), è scritta quasi interamente „na našo“, ma sempre, come negli esempi citati sopra, da intendere come un'ibridazione del dialetto con lo standard linguistico di oltre Adriatico:

„Veloštovani Gospodine

Nemoreš misliti, ni zaćeti kako drago je mi bio imati tvoj listak, ko ja cekahu, kako brižan ceka dažd na mjesac svibanj! Hvala tebi za tvoju dobrotu. 
Žao je mi bio ti ćuti bolestan! Moja žena, moje kceri, moi sin, moi dète, učeniki, rodiaki, molu Boga da ti vernje sdravje bolje od prvo! Bog je milostiv; a on nemore ostati svoje stvorenie.

$[\ldots]$

Nemorem velo ti pisati; da stojim razboljen. Vratajuc iz Lavinu, sunce je mi skodio; i sada gledam postelju. Danas ni je mi došla ognjca, i sutra ću se ustati. Svud di gredeš, nemoj nas zaboraviti! Gledaj! Ja pisam zaboraviti, ni zabiti!!

Zašto ste nas ostali nako ure? Nemorahte zadržati snami drugo mallo vrima?

[...]. Nemoj zaćuditi! Ja pisam, kako goverem“.

La seconda lettera a Drinov, scritta il giorno dopo, è interamente in italiano. La differente scelta del mezzo linguistico ha cause evidenti: mentre i discorsi personali e le cordialità sono affidate da De Rubertis alla freschezza semplice del dialetto, le informazioni di carattere storiografico ed erudito che costituiscono la parte finale della prima e la totalità della seconda lettera richiedono il passaggio all'italiano, lingua di cultura e più maneggevole all'ora di discorsi stilisticamente complessi.

Il dialetto torna, per le medesime ragioni, nella terza ed ultima lettera indirizzata a Drinov nell'estate del 1870. De Rubertis, sconfortato, comunica (in italiano) una serie di gravi lutti familiari: il già ricordato rapimento di un nipote da parte di una banda di briganti e la morte prematura di un altro nipote. L'uso dell'italiano si spiega con la dovizia di dettagli esplicativi e con l'esuberante pathos retorico a cui l'insegnante molisano ricorre per descrivere i fatti. Il dialetto si ripresenta, invece, sotto la forma di un pianto poetico, ancora una volta con l'affiorare di un registro fortemente emotivo e diretto:

„Nimamo već suze da plakati! Plaćiu svizde, plaćiu stine; plaće zemlja di on sada poćinje! Ni to je sve - Je još za plakati!

Ah sì, o Signore, vi ha ancora da piangere!“4

Nel complesso, le lettere di Giovanni De Rubertis a Marin Drinov e Vikentij Makušev rappresentano una pagina minore, ma significativa nella storia della slavistica, come testimonianza culturale e linguistica dei primi passi nello studio scientifico delle comunità slavo-molisane e del loro dialetto. La figura stessa del

\footnotetext{
${ }^{4}$ De Rubertis sta verosimilmente citando una canzone popolare, o un carme di sua stessa composizione.
} 
De Rubertis attende di essere studiata più approfonditamente: mancano a tutt'oggi lavori analitici che facciano il punto sulla vita e sull'attività di questo appassionato paladino della slavitudine molisana, colmando non poche lacune e verificando notizie spesso contraddittorie.

\section{Appendice}

\section{Tre lettere inedite di Giovanni De Rubertis a Marin Drinov 5}

\section{I}

Vodaživa 24 Lipanj 1870

Veloštovani Gospodine

Nemoreš misliti, ni zaćeti kako drago je mi bio imati tvoj listak, ko ja cekahu, kako brižan ceka dažd na mjesac svibanj! Hvala tebi za tvoju dobrotu.

Žao je mi bio ti ćuti bolestan! Moja žena, moje kceri, moi sin, moi dète, učeniki, rodiaki, molu Boga da ti vernje sdravje bolje od prvo! Bog je milostiv; a on nemore ostati svoje stvorenie.

Ako naš Vladika ${ }^{6}$ (Biskup) u Rim je ti primio jako ljepo, ja ni se zaćudio. On je našia krv; i sve što je ćinio za tebe, je vazda mallo ćinio. Ja ću mu pisati na ovu drugu nedilju.

Nemorem velo ti pisati; da stojim razboljen. Vratajuc iz Larinu, sunce je mi skodio; i sada gledam postelju. Danas ni je mi došla ognjca, i sutra ću se ustati. Svud di gredeš, nemoj nas zaboraviti! Gledaj! Ja pisam zaboraviti, ni zabiti!!

Zašto ste nas ostali nako ure? Nemorahte zadržati snami drugo mallo vrima?

Moja obiteljca, i osobito Sindaco velo ti pozdravu; a moi unuk Romualdo Vettin ${ }^{7}$ stoi u Napulu (Strada S. Mattia N.54, terzo piano).

Ti pošaljem moja tumaćenje od piesnica, ka je mi posla Makušev. Nemoj zaćuditi! Ja pisam, kako goverem.

Ćekam drughe goste u Rujan. Reci gnjim da ja od sada jih stisknim u srcu! Na miesac Rujan ni jesam tako jatast ${ }^{8}$.

${ }^{5}$ Come si è detto sopra, le tre lettere sono custodite presso la Biblioteca Nazionale Bulgara a Sofia (Национална Библиотека «Св. Св. Кирил и Методий», НБКМ БИА, ф. 111, арх. ед. 267, л. 1-6).

${ }^{6}$ Il vescovo di Nardò, Luigi Vetta, con cui De Rubertis era imparentato.

${ }^{7}$ Romualdo Vetta. Vettin è la versione slavo-molisana del cognome.

8 Jatast: sic. Non sembra esistere una parola simile nel dialetto di Acquaviva, né, ovviamente esiste nella lingua standard. Tuttavia, appare abbastanza chiaro che De 
Misli ozdraviti. Sada ti stisknim ruku, kako brat, bratu. Sbogom.

Tvoj ponizni sluga

Ivan de Rubertis

P.S. Non saprei dirti se gli Slavi fossero venuti Cattolici o si fossero in seguito cattolicizzati. Solo leggesi nell'opera voluminosa di Monsignor Tria, Vescovo di Larino, ${ }^{9}$ che a' suoi tempi gli Albanesi avevano ancora rito Greco; e che l'ultimo arciprete di rito Greco fu un tal Peta di Portocannone (paese albanese). Ma degli Slavi? Nulla Tria ne dice; forse perché le colonie Slave non appartenevano alla sua Diocesi.

Canzonetta Popolare Slava

Versione

È bello sorgere

In sul mattino,

Quando l'Aurora

Il Mondo indora,

E il canto ascoltavi

Dell'usignuol.

Ah l'usignuolo

Saltella solo,

Sul far del giorno

Al pozzo intorno

Sciogliendo tenere

Note di duol.

Sul pozzo miravi

Un bianco foglio.

Sovr'esso i rai

Volgi, e vedrai,

Che a nere lettere

Tal scritto appar.

È colpa amare

Una donzella!

È colpa amarla,

E poi lasciarla;

Al seno stringerla,

E lei scordar.

Rubertis la forma come variante di 'jadan' (*jadast), e il senso risulta perciò piuttosto chiaro: a settembre non sarà troppo occupato.

9 II riferimento è a Giovanni Andrea Tria, Memorie Storiche Civili, ed Ecclesiastiche della città, e Diocesi di Larino, Roma, 1744. 
Son d'una vergine

Gravi le lagrime!

Quando sospira,

Dio l'ode, e mira!

Se impreca, trema

La terra allor.

Una sua lagrima

Se a terra gronda,

Si addentra e forma

Fossa profonda

Tre lance, e larga

Ben quattro ancor.

Tri duboko, ćetiri široko!! ${ }^{10}$

II

[Acquaviva 25 giugno 1870]

Onorevole Signore,

leri le scrissi una mia lettera in lingua Slava; sia per appagare un suo desiderio, sia per darle un saggio del nostro dialetto. Oggi le torno a scrivere per alcune delucidazioni; e nel tempo istesso le invio un numero dell'Indipendente, in cui si parla di Lei, del Signor Makušev e delle Colonie Slave.

Nella penultima strofa della mia versione si è incorso (per la fretta) in un errore, che bisogna emendare. Dove leggesi:

Son delle vergini, ec.

la prego correggere nel seguente modo:

Son d'una vergine, ec.

Ne interesso su tal riguardo la sua bontà.

Errata corrige

Nell'articoletto di risposta al suo quesito, se cioè gli Slavi fossero venuti Cattolici, o si fossero in seguito Cattolicizzati, io le faceva conoscere che Monsignor Tria, Vescovo di Larino, asseriva che gli Albanesi, a' suoi tempi, avevano ancora rito Greco, ma che non faceva parola degli Slavi, perché forse non appartenenti alla sua Diocesi. Ciò deve intendersi solamente a quanto riguarda il rito Greco, o Cattolico; perché Monsignor Tria parla ancora delle colonie Slave. Che anzi nel riportare una capitolazione delle colonie Albanesi di Ururi, asserisce che questa capitolazione venne segnata da un tal Andreas de Comte alias Glava: ora questo

${ }^{10}$ Si tratta di una versione della „canzonetta“ popolare Djevojačka kletva. Probabilmente De Rubertis si ispirava al testo pubblicato nel 1847 da Antun Brlić (Djevojačka kletva. Kolo, in Članci za literaturu, umětnost i narodni život. Ur. Stanko Vraz, knj.V, Tiskarnja Matice Ilirske u Zagrebu, 1847, p. 55). 
glava è un vocabolo slavo, che significa capo. Il che sempre più confirmerebbe che Albanesi e Slavi convivevano insieme, e che la loro emigrazione fu contemporanea. Ma per quanto costa che gli Albanesi avessero rito Greco, altrettanto è incerto se le colonie Slave avessero rito Greco, o Latino.

Ritornando a Napoli, interesso la gentilezza del suo animo a rimettermi una sua fotografia. Me l'ha rimessa il Signor Makušew; è necessario che me ne faccia un dono anche il Signor Drinow; affinché io potessi arricchire la mia Illirica Pinacoteca. Forse sono troppo esigente; ma la prego compatirmi in grazia della stima che le professo.

Tutti i miei la salutano distintamente; ed io stringendola al cuore, mi pregio di essere

Acquaviva, 25 Giugno 1870

Suo amico vero

Giovanni de Rubertis

III

Acquaviva, 11 Agosto 1870

Onorevole Signore

Grata la sua lettera; e gratissimo il suo dono, che serberò per sua memoria. Solo mi dispiace di non aver fatto profondi studi su' caratteri cirillici; ma la sua opera mi spingerà forse a riprenderli con amore.

Gravi infortuni, ineffabili sventure opprimono, e straziano il mio animo, e quello de' miei congiunti! Non ha guari una mia sorella, colpita da apoplessia, moriva repentinamente, lasciandoci in preda alla più viva desolazione! Nella scorsa settimana un mio nipote, Francesco Vetta, Cavaliere della Corona d'Italia, Ispettore Capo della Questura di Caserta, e fratello germano del Padre Romualdo, terminava miseramente la sua gloriosa carriera, nell'età di 34 anni, quando la vita è ancora una speranza!

Quale orribile sventura! Egli moriva per grave caduta, mentre saliva sul convoglio ferroviario, nell'atto che era in movimento, nella stazione di Caserta! Oh Dio! Una vita sì preziosa, sì splendida di lieto avvenire, si è spenta per sempre!! Nimamo već suze za plakati! Plaćiu svizde, plaćiu stine; plaće zemlja di on sada poćinje! Ni to je sve - Je još za plakati!

Ah sì, o Signore, vi ha ancora da piangere! Nel giorno due di questo mese un altro mio nipote, il Signor Carlo Leone di Guglionesi, Cognato del Padre Romualdo, è stato catturato da' briganti, i quali pretendono pel suo riscatto l'ingente somma di cinquanta mila lire!! Pendono le trattative (trattative con $\mathrm{i}$ briganti!!) e si farà qualunque sagrificio, onde salvare un infelice padre di 
numerosa famiglia. Ella mi scuserà se ho osato metterla a parte delle nostre afflizioni! Oramai dobbiamo riguardarci non solo come amici, ma come fratelli!

II Signor Makušev mi scrisse una lettera in lingua Slava, ringraziandomi delle raccomandazioni presso il Prefetto di Ancona Sig.r Nicola de Luca, mio congiunto, ed amico. II Prefetto lo accolse con tutti i riguardi dovuti a' suoi meriti; e l'istesso Sig.r Makušev si esprimeva così: "Vidio sam G. de Luca, koj primio mi je jako ljepo. Ja se zaćudio, kad on mi je kazao, da je iz onih vaših poseobini[a], koje imao sam sreću posjetiti zajedno s mojim prijateljom Drinovim ec. ... Vale a dire in buon Italiano "Io mi sono meravigliato quando Egli mi disse di essere di quelle vostre Colonie, che io ebbi la sorte di visitare col io amico Drinov". A queste parole un amaro sorriso sfiorò le mie labbra, perché il Prefetto De Luca, sebbene fosse delle nostre contrade, non appartiene alle nostre Colonie. Su questo riguardo io risposi con termini vaghi; perché non mi conveniva disingannare il Sig. Makušev, prima di scrivere al Prefetto suddetto, onde conoscere in qual senso abbia Egli profferito quelle parole. Non vorrei che questo incidente, di cui non sono affatto colpevole, valesse a farmi perdere la stima del Signor Makušev, o atterrare le nostre amichevoli relazioni. Dico questo, perché Egli, abbandonando Ancona, non mi mise a parte del suo nuovo indirizzo. ${ }^{11}$ Se lo vedrà, interesso la bontà del suo animo a fargli leggere questo articoletto. Se il prefetto ha mentito, è forse mia la colpa? Chi poteva prevederlo?

Nel prossimo Ottobre sarò in Napoli, ed in Roma, ed allora le rimetterò la mia fotografia.

Tutti i giornali di Napoli piangono la morte di mio Nipote. Legga il Pungolo del giorno sette, o otto, vegga qual perdita siasi fatta! Addio

Suo amico vero

Gio. de Rubertis

${ }^{11}$ I contorni della questione rimangono imprecisi: Makušev era giunto ad Ancona con una lettera di raccomandazione del De Rubertis per il prefetto De Luca, suo "congiunto", cioè parente acquisito. La raccomandazione gli era risultata utile per lavorare negli archivi anconetani e per ricevere un'accoglienza calorosa da parte del De Luca (Aloe, 2013). Tuttavia, al partire da Ancona alla volta di Bologna, lo storico russo smise di rispondere al De Rubertis e fece, per così dire, perdere le sue tracce. De Rubertis temeva che ciò fosse legato alla bugia del prefetto che, contraddicendo quanto lui aveva anticipato a Makušev, si era dichiarato, senza esserlo, uno slavo molisano. Tuttavia, la spiegazione appare quantomeno esagerata. Più probabilmente, Makušev aveva perso interesse per le colonie molisane e per l'amicizia un po' troppo soffocante di Giovanni De Rubertis. 


\section{Bibliografia}

Stefano Aloe, »ll primo viaggio slavistico in Italia: i percorsi dello storico russo Vikentij Makušev (1869-1871)«, in: Viaggi e viaggiatori nell'Ottocento. Itinerari, obiettivi, scoperte (a cura di Marco Severini), Marsilio, Venezia 2013, 115-127.

Giovanni De Rubertis, „Delle colonie Slave nel Regno di Napoli: Lettere del professore Giovanni De Rubertis«, Zara. L'Osservatore Dalmata (estratto), 1856.

Cristo Kovačić, Gli Slavi serbi dell'Italia, Cherubini, Ancona 1884.

Stjepan Krpan, »De Rubertis, Giovanni (Ivan)«, in: Hrvatski biografski leksikon, III. Leksikografski zavod Miroslav Krleža, Zagreb 1993., 211-212.

Milan Rešetar, Die Serbo-Kroatischen Kolonien Süditaliens, Wien. Alfred Hölder 1911 (trad. it.: Le colonie serbocroate nell'Italia meridionale, a cura di Walter Breu e Monica Gardenghi. Campobasso [Amministrazione provinciale] 1997).

Стефано Алоэ, "Джованни Де Рубертис и другие итальянские корреспонденты В.В. Макушева«, Slavica litteraria, X, 15/2012, Supplementum 2: Развитие славистики в зеркале эпистолярного наследия и других личных документов = Vývoj slavistiky v zrcadle epistulárního dědictví a jiných osobních dokumentů (eds. Sergio Bonazza, Ivo Pospíšil). Brno 2012, 11-29.

Иван Де Рубертис, „Славянские поселения в Неаполитанском королевстве. Письма тамошнего учителя Иована де Рубертисщ, in: Чтения при Имп. Обществе истории и древностей российских при Моск. ун-те, январь-март 1858, кн. І, 3-29.

Александр Д. Дуличенко, "Диалект молизских славян Италии и русская славистика XIX - начала XX вв.", in: Из истории славяноведения в России. Труды по русской и славянской филологии (Уз Тартуского Гос. Ун-та, вып. 573), Тартуский Гос. Университет, Тарту 1981, 95109.

Ристо Ковачић, „Српске насеобине у Јужној Италијищ, Гласник Српског Ученог Друштва, 62, 1885., 273-340.

Людмила П. Лаптева, История славяноведения в России в ХІХ веке. Индрик, Москва 2005.

Викентий В. Макушев, „О славянах Молизского графства в Южной Италии«, Записки Имп. Академии Наук, 1871, т. 18, кн. 1., 1871, 3156.

Викентий В. Макушев, Десять месяцев за границею. Путевые заметки и наблюдения. <1881>. Отдел Рукописей РГБ, ф. 156. оп. 4. д. 7. л. $16,1881$. 
Лиляна Минкова, »Переписка Викентия В. Макушева с Марином Ст. Дриновым 1869-1878 гг.«, Bulgarian historical review, № 1-2., 1981, 180-213.

Лиляна Минкова, „В.В. Макушев и становление болгарской исторической наукищ, in: Aktuálne problémy dejín slavistiky: materiály zasadnutia Medzinárodnej komisie pre dejiny slavistiky (Smolenice 11.-14. február 1985), (Pripravili Vladimír Matula a Tatiana Ivantyšynová), Slovenská akadémia vied - Ústav historických vied, Bratislava 1981, 161-175.

Лиляна Минкова, „Кореспонденцията на Марин Дринов с Викентий Василиевич Макушев като извор за историята на българоиталианските и руско-италианските научни връзки през XIX век", in: България, Италия и Балканите: Обществено-исторически и културно-естетически взаимоотношения XV-XX век (Под ред. Георги Димов), БАН, София 1988, 179-185.

Майя В. Никулина, »Письма М. С. Дринова В. В. Макушеву (1870-1875 гг.)«, Советское славяноведение, № 2, 1974, 64-69.

Никша Стипчевић et alii (уред.), Грађа за биографски речник чланова Друштва Српске Словесности, Српског Ученог Друштва и Српске Краљевске Академије (1841-1947), Српска Академия наука и уметности, Београд 2007.

\section{Riassunto}

I riscontri dei primi slavisti che nella seconda metà dell'Ottocento visitarono le colonie slavo-meridionali del Molise sono di interesse per la storia della slavistica, e in particolare per lo sviluppo degli studi slavistici internazionali legati all'Italia. La slavistica internazionale non aveva ancora sviluppato un interesse specifico per l'Italia. Fondamentali per questa scoperta, gravida di risultati, furono l'attività divulgativa dello storico slavo-molisano Giovanni De Rubertis e il viaggio ad Acquaviva Collecroce di due storici slavi, il russo Vikentij Makušev e il bulgaro Marin Drinov nel 1870. La corrispondenza epistolare fra i due eminenti studiosi slavi e I'entusiasta storico dilettante di Acquaviva Collecroce, insieme ad una pagina del Diario di Makušev e ad altri materiali inediti o di difficile reperimento, permettono di trarre considerazioni sulla coscienza etno-linguistica degli slavi molisani nella seconda metà dell'Ottocento e sui loro orientamenti culturali. 


\section{Marin Drinov and Vikentij Makušev's journey to Molise in the letters of Giovanni De Rubertis}

(Summary)

The first travel for Slavistic purposes to the Slavic speaking colonies settled in the Southern Italian region of Molise was pursued by the two historians Vikentij Makushev (Russia) and Marin Drinov (Bulgaria) in the spring of 1870 . In the small village Acquaviva Collecroce, the two scholars were enthusiastically welcomed by Giovanni De Rubertis - the promoter of the Molisean Slavic identity rebirth in the area -, whose support and friendly collaboration happened to be fruitful even after their departure. Thanks to the epistolary correspondence between the eminent scholars and the Italian amateur, and the rare documents recently discovered, it is possible to debate the ethno-linguistic awareness of the Molisean Slavic people in the second half of the $19^{\text {th }}$ century, as well as their cultural tendencies and the main Slavic influences they absorbed indirectly, before the connections with the other shore of the Adriatic Sea were established.

Keywords: History of Slavistics, Molisean Slaves, Giovanni De Rubertis, Vikentij Makušev, Marin Drinov, Acquaviva Collecroce 